\begin{tabular}{l|l|l}
\hline 1894 & $\Delta \alpha$ & $\Delta d$ \\
\hline
\end{tabular}

(I 3) Amalthea.

[A. N. $3^{2} 38$ ].

\begin{tabular}{ll|l|l} 
Agos. & +0.40 & +1.5 \\
Sett. & 4 & +0.70 & +6.7 \\
& 7 & +0.39 & +6.5
\end{tabular}

\begin{tabular}{|l|l|l}
\hline $\mathrm{1} 894$ & $\Delta \alpha$ & $\Delta \delta$ \\
\hline
\end{tabular}

(87) Sylvia.

[B. A. J. 1896].

Nov. $3|-0.77|-5$ " t

$$
\begin{array}{l|l|l}
5 & -0.44 & -4.5
\end{array}
$$

(76) Freia.

[B. A. J. 1896].

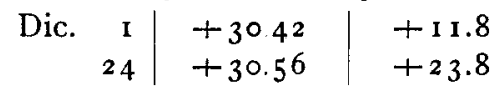

\begin{tabular}{c|c|c}
\hline 1894 & $\Delta \alpha$ & $\Delta \delta$ \\
\hline \multicolumn{3}{c}{$(164)$ Eva. }
\end{tabular}

[B. A. J. 1896 ].

Dic. $24\left|+2^{5} 85\right|+14.9$

$$
\begin{array}{l|l|l}
25 & +3.19 & +17.8
\end{array}
$$

(12I) Hermione.

[B. A. J. 1896 ].

Dic. \begin{tabular}{l|l|l}
26 & +7.45 & +35.5 \\
28 & +7.06 & +34.1
\end{tabular}
Queste osservazioni fanno seguito a quelle già pubblicate nel numero 3257 delle A. $\mathrm{N}$.

Furono fatte tutte in campo oscuro col micrometro a larghe lamine, tenendo conto nell' osservazione dei passaggi ordinariamente soltanto delle sparizioni. Di solito un confronto in ascensione retta comprende le due differenze fornite dai tempi dei passaggi alle due lamine; un confronto in declinazione comprende quattro differenze micrometriche.

Padova, Osservatorio Astronomico, 1895 Gennaio 9.
Con 16 serie di osservazioni su pianeti diversi calcolai altrettanti valori dell' errore probabiie di una differenza di ascensione retta e di una differenza di declinazione; dal loro medio risulto per errore probabile della differenza di ascensione retta ottenuta con 8 confronti (I 6 puntate) $0.035 \pm 0.003$, e per errore probabile della differenza di declinazione avuta da 4 confronti ( 16 puntate) $0.42 \pm 0 " 03$.

\title{
Ueber die Beziehungen verschiedener Methoden zur Untersuchung der Bewegung des Sonnensystems.
}

Von H. Kobold.

Aus den Ausdrücken der drei rechtwinkligen auf den als ruhend betrachteten Sonnenmittelpunkt als Coordinatenanfang bezogenen Coordinaten eines Sterns

$$
x=\varrho \cos \alpha \cos \delta \quad y=\varrho \sin \alpha \cos \delta \quad z=\varrho \sin \delta
$$

ergeben sich durch Differentiation die drei Ausdrücke :

$$
\begin{aligned}
& \mathrm{d} x=-\rho \cos \delta \sin \alpha \mathrm{d} \alpha-\rho \sin \delta \cos \alpha \mathrm{d} \delta+\cos \delta \cos \alpha \mathrm{d} \rho \\
& \mathrm{d} y=\quad \rho \cos \delta \cos \alpha \mathrm{d} \alpha-\rho \sin \delta \sin \alpha \mathrm{d} \delta+\cos \delta \sin \alpha \mathrm{d} \rho \\
& \mathrm{d} z=\quad \quad \quad \cos \delta \mathrm{d} \delta+\sin \delta \mathrm{d} \rho
\end{aligned}
$$

und aus diesen durch Elimination

$$
\begin{aligned}
\cos \delta \mathrm{d} \alpha & =-\sin \alpha \frac{\mathrm{d} x}{\varrho}+\cos \alpha \frac{\mathrm{d} y}{\varrho} \\
\mathrm{d} \delta & =-\cos \alpha \sin \delta \frac{\mathrm{d} x}{\varrho}-\sin \alpha \sin \delta \frac{\mathrm{d} y}{\varrho}+\cos \delta \frac{\mathrm{d} z}{\varrho} \\
\mathrm{d} \rho & =\cos \alpha \cos \delta \mathrm{d} x+\sin \alpha \cos \delta \mathrm{d} y+\sin \delta \mathrm{d} z
\end{aligned}
$$

\section{G. Ciscato.}

so entstehen die Gleichungen

$$
\xi=q \cos A \cos D \quad \eta=q \sin A \cos D \quad \zeta=q \sin D
$$

$$
\begin{aligned}
\cos \delta \mathrm{d} \alpha & =\frac{q}{\varrho} \cos D \sin (\alpha-A)-\sin \alpha \frac{\mathrm{d} x}{\varrho}+\cos \alpha \frac{\mathrm{d} y}{\varrho} \\
\mathrm{d} \delta & =\frac{q}{\varrho} \sin \delta \cos D \cos (\alpha-A)-\frac{q}{\varrho} \cos \delta \sin D-\cos \alpha \sin \delta \frac{\mathrm{d} x}{\varrho}-\sin \alpha \sin \delta \frac{\mathrm{d} y}{\varrho}+\cos \delta \frac{\mathrm{d} z}{\varrho} \\
\mathrm{d} \varrho & =-q \cos \delta \cos D \cos (\alpha-A)-q \sin \delta \sin D+\cos \alpha \cos \delta \mathrm{d} x+\sin \alpha \cos \delta \mathrm{d} y+\sin \delta \mathrm{d} z .
\end{aligned}
$$

In diesen Gleichungen sind nun $\mathrm{d} x, \mathrm{~d} y, \mathrm{~d} z$ die sogenannten motus peculiares der Fixsterne und wir sehen also, dass, wenn wir die beiden ersten Gleichungen zur Bestimmung der Werthe $A, D$ benutzen, wie es in der nach Airy benannten Methode geschieht, wir die Voraussetzung machen müssen 


$$
\Sigma\left(\sin \alpha \frac{\mathrm{d} x}{\rho}-\cos \alpha \frac{\mathrm{d} y}{\rho}\right)=0
$$

$\Sigma\left(\cos \alpha \sin \delta \frac{\mathrm{d} x}{\varrho}+\sin \alpha \sin \delta \frac{\mathrm{d} y}{\rho}-\cos \delta \frac{\mathrm{d} z}{\rho}\right)=0$

Bestimmen wir dagegen dieselben Grössen aus der dritten Gleichung mit Hülfe der Bewegungen im Visionsradius, so ist die nothwendige Voraussetzung

$$
\Sigma(\cos \alpha \cos \delta \mathrm{d} x+\sin \alpha \cos \delta \mathrm{d} y+\sin \delta \mathrm{d} z)=0
$$

Nun ist aber :

$$
-\sin \alpha \frac{\mathrm{d} x}{\rho}+\cos \alpha \frac{\mathrm{d} y}{\rho} \quad \begin{aligned}
& \text { die Componente der motus peculiares für die Richtung des Parallels in } \\
& \text { Bogenmaass; }
\end{aligned}
$$

$-\cos \alpha \sin \delta \frac{\mathrm{d} x}{\varrho}-\sin \alpha \sin \delta \frac{\mathrm{d} y}{\varrho}+\cos \delta \frac{\mathrm{d} z}{\varrho}$ die Componente der motus peculiares für die Richtung des Declinationskreises in Bogenmaass;

$\cos \alpha \cos \delta \mathrm{d} x+\sin \alpha \cos \delta \mathrm{d} y+\sin \delta \mathrm{d} z$ die Componente der motus peculiares für die Richtung des Visionsradius in linearem Maass.

Nimmt man nun an, dass die motus peculiares für die Richtungen der Coordinatenaxen wie zufällige Fehler zu behandeln sind, so sind auch diese Componenten in derselben Weise zu behandeln, und man hat daher angenommen, dass man für die Bestimmung der Sonnenbewegung ibren Einfluss vernachlässigen könne. Airy geht bei der Ableitung seiner Gleichungen aus von der Annahme, dass die Vertheilung der Sterne so gleichförmig sei, dass man alle Summen, deren einzelne Glieder $\sin \alpha$ oder $\cos \alpha$ als Factor enthalten, als verschwindend betrachten könne. Dies ist im Allgemeinen jedenfalls nicht gestattet. Bildet man z. B. für die 1400 Bradley'schen Sterne, die ich in Nr. $316_{3}$ benutzt habe, die Summen $\cos \alpha \cos \delta$ bez $\sin \alpha \cos \delta$, so ergeben sich folgende Zahlen

$\begin{array}{cccc}\text { Classe } & \text { Zahl d. Sterne } & \Sigma \cos \alpha \cos \delta & \Sigma \sin \alpha \cos \delta \\ \text { I } & 24 & -3.35 & -1.3^{2} \\ 2 & 43 & -5.15 & +2.12 \\ 3 & 101 & -15.58 & +9.31 \\ 4 & 210 & -29.14 & +12.15 \\ 5 & 386 & -51.76 & +21.61 \\ 6 & 636 & -80.35 & +48.51\end{array}$

Ein ähnliches, offenbar durch die ungleiche Vertheilung der Sterne veranlasstes Verhalten, werden jedenfalls auch die anderen Functionen von $\alpha$ zeigen und es wird daher der Einfluss eines systematischen Verhaltens der motus peculiares für die einzelnen der drei Bedingungsgleichungen ein verschiedener sein. Es ist auch sehr wohl möglich, dass die motus peculiares in der zum Visionsradius senkrechten Ebene sich der Annahme entsprechend verhalten, während dies für die Richtung des Visionsradius nicht der Fall ist. So würde es sich ganz ungezwungen erklären, dass die beiden erwähnten Methoden der Bestimmung der
Sonnenbewegung zu mit einander nicht zu vereinigenden Resultaten führen.

Die geringe Anzahl der von Vogel (A. N. 3150 ) be. nutzten Sterne halte ich nicht für einen ausreichenden Grund zur Erklärung des Unterschiedes. Denn behandelt man dieselben 5 r Sterne nach der Airy'schen Methode, um den Ort des Apex aus den Bewegungen in der zur Gesichtslinie senkrechten Ebene zu bestimmen, so ergiebt sich folgender Werth :

$$
A=247^{\circ} \mathrm{I}^{\prime} \quad D=47^{\circ} 56^{\circ} \quad q=\text { 0.' I9 I }
$$

während Vogel findet

$$
A=206: \text { r } \quad D=45^{\circ} \text {. }
$$

Hierbei ist $q$ in demselben Maasse ausgedrückt, wie die beobachteten Bewegungen, es bedeutet also die jährliche Eigenbewegung der Sonne gesehen aus der mittleren Entfernung der 5 I Sterne, da eine Hypothese über die Grösse $\varrho$ bei der Rechnung nicht eingeführt ist. Der berechnete Werth entspricht dem bisher angenommenen genügend, um zu beweisen, dass die behandelten 5 I Sterne sich nicht wesentlich anders verhalten, wie die Mehrzahl aller Sterne.

Führen wir in die Ausdrücke für $\mathrm{d} \alpha \cos \delta$ und $\mathrm{d} \delta$ die Bewegung $\mathrm{d} s$ im Bogen grössten Kreises und ihre Richung $\varphi$ ein, nennen weiter $A_{0}$ und $D_{0}$ Näberungswerthe der Coordinaten des Apex, aus denen wir den Abstand vom Antiapex $\Delta$ und die Richtung auf denselben $\psi$ finden durch die Gleichungen

$$
\begin{aligned}
\sin \Delta \sin \psi & =\cos D_{0} \sin \left(\alpha-A_{0}\right) \\
\sin \Delta \cos \psi & =-\sin D_{0} \cos \delta+\cos D_{0} \sin \delta \cos \left(\alpha-A_{0}\right) \\
\cos \Delta & =-\sin D_{0} \sin \delta-\cos D_{0} \cos \delta \cos \left(\alpha-A_{0}\right)
\end{aligned}
$$

so ergeben sich die Differentialgleichungen

$$
\begin{aligned}
\mathrm{d} s \cdot \sin \varphi & =\frac{q}{\varrho} \sin \Delta \sin \psi-\frac{q}{\varrho} \sin D_{0} \sin \left(\alpha-A_{0}\right) \mathrm{d} D-\frac{q}{\varrho} \cos D_{0} \cos \left(\alpha-A_{0}\right) \mathrm{d} A \\
\mathrm{~d} s \cdot \cos \varphi & =\frac{q}{\varrho} \sin \Delta \cos \psi-\frac{q}{\varrho}\left(\sin \delta \sin D_{0} \cos \left(\alpha-A_{0}\right)+\cos \delta \cos D_{0}\right) \mathrm{d} D+\frac{q}{\varrho} \sin \delta \cos D_{0} \sin \left(\alpha-A_{0}\right) \mathrm{d} A .
\end{aligned}
$$

Aus beiden Gleichungen folgt weiter:

$$
\begin{aligned}
\mathrm{d} s \sin (\varphi-\psi)= & -\mathrm{d} A \frac{q}{\varrho}\left(\cos D_{0} \cos \left(\alpha-A_{0}\right) \cos \psi+\sin \delta \cos D_{0} \sin \left(\alpha-A_{0}\right) \sin \psi\right) \\
& -\mathrm{d} D \frac{q}{\varrho}\left(\sin D_{0} \sin \left(\alpha-A_{0}\right) \cos \psi-\sin \delta \sin D_{0} \cos \left(\alpha-A_{0}\right) \sin \psi-\cos \delta \cos D_{0} \sin \psi\right)
\end{aligned}
$$


Ist $\varphi-\psi$ ein kleiner Winkel, so dass wir den Sinus mit dem Bogen vertauschen dürfen und drücken wir $\mathrm{d} A$ und $\mathrm{d} D$ in Bogenmaass aus, so erhalten wir durch Einführung der vorhin gegebenen Ausdrücke von sin $\psi$ und $\cos \psi$ die Gleichung

$$
\mathrm{d} s \cdot(\varphi-\psi)=\frac{q}{\rho} \frac{\mathrm{I}}{\sin \Delta}\left(\sin D_{0} \cos \delta \cos \left(\alpha-A_{0}\right)-\cos D_{0} \sin \delta\right) \cos D_{0} \mathrm{~d} A+\frac{q}{\rho} \frac{\mathrm{I}}{\sin \Delta} \cos \delta \sin \left(\alpha-A_{0}\right) \mathrm{d} D
$$

Diese Gleichung beruht also auf denselben Annahmen wie die Grundgleichungen der Airy'schen Methode, sie verlangt bezüglich der motus peculiares ganz das gleiche wie jene, und sie wird also auch bei ihrer Anwendung zu denselben Werthen der Coordinaten des Apex führen müssen. Fassen wir jetzt aber die motus peculiares auf als zufällige, die beobachteten Eigenbewegungen entstellende Fehler, dann wäre $\mathrm{d} s$ die Bewegung der Sonne gesehen von dem betreffenden Stern aus und es besteht die Relation

$$
\mathrm{d} s=\frac{q}{\varrho} \sin \Delta
$$

und dieses ergiebt in die vorige Gleichung eingesetzt, die neue Bedingungsgleichung

$$
\varphi-\psi=\frac{\mathbf{I}}{\sin ^{2} \Delta}\left(\sin D_{0} \cos \delta \cos \left(\alpha-A_{0}\right)-\cos D_{0} \sin \delta\right) \cos D_{0} \mathrm{~d} A+\frac{\mathrm{I}}{\sin ^{2} \Delta} \cos \delta \sin \left(\alpha-A_{0}\right) \mathrm{d} D
$$

Dies ist nun aber die Bedingungsgleichung der Argelander'schen Methode, abgesehen von der Gewichtsbestim. mung, die auf der rechten Seite noch den Factor $\sin A$ hinzufügt.

Aus dieser Ableitung der Gleichung ergiebt sich jetzt aber, dass die Argelander'sche und die Airy'sche Methode auf denselben Voraussetzungen bezüglich der motus peculiares der Sterne beruhen, so dass die Uebereinstimmung der Resultate beider Rechnungsmethoden eine nothwendige ist

und bezüglich der Richtigkeit jener Voraussetzungen nichts zu beweisen vermag.

Die Ausdrücke für $\mathrm{d} x, \mathrm{~d} y, \mathrm{~d} z$, von denen wir ausgingen, können zur Bestimmung der Gesammtbewegung benutzt werden für alle diejenigen Sterne, für welche wir die Parallaxe und die Bewegung im Visionsradius kennen. Nennen wir $k$ den Quotienten: Erdbahnhalbmesser, ausgedrückt in derselben Einheit wie d $\varrho$, dividirt durch die Zahl der Zeitsecunden, die auf ein Jahr gehen, so werden die Endgleichungen

$$
\begin{aligned}
\mathrm{d} x & =-k \cos \delta \sin \alpha \frac{\mathrm{d} \alpha}{\pi}-k \sin \delta \cos \alpha \frac{\mathrm{d} \delta}{\pi}+\cos \delta \cos \alpha \mathrm{d} \rho \\
\mathrm{d} y & =k \cos \delta \cos \alpha \frac{\mathrm{d} \alpha}{\pi}-k \sin \delta \sin \alpha \frac{\mathrm{d} \delta}{\pi}+\cos \delta \sin \alpha \mathrm{d} \rho \\
\mathrm{d} z & =k \cos \delta \frac{\mathrm{d} \delta}{\pi}+\sin \delta \mathrm{d} \rho
\end{aligned}
$$

In einer demnächst in den Nova Acta der Leopoldina erscheinenden Abhandlung habe ich diese Ausdrücke auf die Sterne angewandt, die zur Zeit der Untersuchung unterworfen werden können. Bezüglich der Resultate verweise ich auf jene Abhandlung, möchte hier aber die Gelegenheit benutzen darauf hinzuweisen, dass eine Bestimmung der

Parallaxe der von Vogel benutzten Sterne mit gleicher Genauigkeit, wie sie Elkin in den vorzùglichen New.Havener Messungen erreicht hat, sehr geeignet erscheint, unsere Kenntniss über die wahre Bewegung unserer Sonne und ihr Verhältniss zu den benachbarten Sternen wesentlich zu fördern.

$$
\text { Strassburg I } 895 \text { Jan. } 3 \text { I. }
$$

\section{H. Kobold.}

\section{Beobachtungen des Mars in der Nähe der 1894 Oct. 20 stattgehabten Opposition} angestellt am Pulkowaer Verticalkreise von A. Iwanow.

Wegen des schlechten Wetters gelang es mir nur elf Beobachtungen des Planeten Mars in der Nähe der Opposition zu erhalten. Ich bielt es für nützlich, auch die Declinationen der Sterne zu bestimmen, welche im Nautical Almanac gegeben sind, um mit dem Planeten Mars zusammen beobachtet zu werden. Diese Sterne sind : $\varepsilon$ Piscium, 96 Piscium, o Piscium, $\xi_{1}$ Ceti und $\xi_{2}$ Ceti.

Wenn der Zustand des Himmels es erlaubte, wurden beide Ränder des Mars beobachtet.

Alle gegebenen Grössen, welche nöthig sind, um die Beobachtungen des Mars zu reduciren, sind dem Nautical Almanac entnommen. Die Reduction auf den Meridian wurde mittels der Formeln berechnet, welche in meiner Abhandlung »Observations des planètes faites au grand cercle vertical de Poulkovo (Bull. de l'Académie des Sciences de St. Pét. I 894 , No. 3, Nov.) angeführt sind. Zur Ableitung der Correctionen wegen unvollständiger Beleuchtung der Planetenscheibe wurden die Formeln benutzt, welche Chauve. net in dem zweiten Bande seines Lehrbuchs giebt.

Hierbei muss ich bemerken, dass ich in meiner eben citirten Abhandlung diese Correctionen aus Unachtsamkeit mittels der Formeln berechnete, welche in den Washington Observations gegeben und für den Planeten Mars nicht richtig sind. Die Resultate sind daher um o." 2 bis o."3 fehlerhaft.

Die scheinbaren Oerter der Sterne $\varepsilon$ Piscium, $o$ Piscium und $\xi_{2}$ Ceti sind dem Nautical Almanac und die scheinbaren Oerter des Sterns $\xi_{1}$ Ceti der American Ephemeris entnommen. 